\title{
The use of block maxima method of extreme value statistics to characterise blood glucose curves
}

\author{
Mátyás Szigeti \\ Physiological Controls Research Center \\ Óbuda University \\ Budapest, Hungary \\ szigeti.matyas@phd.uni-obuda.hu
}

\author{
Tamás Ferenci \\ Physiological Controls Research Center \\ Óbuda University \\ Budapest, Hungary \\ ferenci.tamas@nik.uni-obuda.hu
}

\author{
Levente Kovács \\ Physiological Controls Research Center \\ Óbuda University \\ Budapest, Hungary \\ kovacs.levente@nik.uni-obuda.hu
}

\begin{abstract}
In contrast to regular statistics where the focus is on the most typical part of the data and the used metrics are describing that part (usually with the mean or median and variance and interquartile range) where most of the observations came from, there is a branch of statistics which focuses on the extreme events, i.e, the tails of the distributions. These are not simple outliers, like data entry errors, but real part of the data which are far from the central tendency and occur rarely, yet, have relevance and impact. Thus, in many application, they can't be simply neglected. The use of extreme value statistics allows us to fit models on this part of the data and like "regular" statistics, enables us to calculate estimates and predictions, but in this case for extreme values. These methods are frequently used in fields like meteorology and finance where the extreme events have large impact despite their rarity. Because of this rarity, however, only a small fraction of the data can be used so much higher sample size is required for such analysis.

This factor limited the use of extreme value statistics in biomedical field where available technology and costs are strong limitations at frequently measuring most of the biomarkers until recently.

Blood glucose level is one of the exceptions nowadays, as with recent advancements it can be monitored for relatively long time and with high frequency for a patient. Additionally, extreme values of blood glucose levels (both high and low) are associated with - chronic or acute - complications of diabetes.

This paper aims to demonstrate that the use of extreme value statistics, in particular the block maxima approach could be a possible way to characterize blood glucose curves. In addition to providing a metric for the state of the patient and therefore hopefully the associated risks, it allows the comparison of the performance of artificial pancreas systems.

Block maxima method was used to model extreme values of a dataset containing measurements of a single patient with 476 complete days of data acquired with sampling frequency of 15 minutes. Probabilities for exceeding the clinically relevant levels of $270 \mathrm{mg} / \mathrm{dl}$ (cognitive symptoms expected) and $600 \mathrm{mg} / \mathrm{dl}$ (diabetic hyperosmolar syndrome) were calculated and were $3.47 \%$ and $4.96 \cdot 10^{-7} \%$ respectively. Through these estimates it is possible to characterise each patient's status and compare different controllers.
\end{abstract}

Index Terms - extreme value statistics, artificial pancreas, diabetes, block maxima, generalised Pareto distribution, peak over threshold, cgms

Project no. 2019-1.3.1-KK-2019-00007. has been implemented with the support provided from the National Research, Development and Innovation Fund of Hungary, financed under the 2019-1.3.1-KK funding scheme. This project has received funding from the European Research Council (ERC) under the European Union's Horizon 2020 Research and Innovation Programme (Grant Agreement no. 679681).

\section{INTRODUCTION}

Classically, statistics focuses on the analysis of a sample taken from a population in order to draw conclusions about the population. We could describe a population by giving all of its elements but in almost all cases that would be impossible to handle as it would be to much data, or, even more typically, the population is infinite, given by only its distribution. Usually our interest is focused to the most relevant part of the population which are often the most common elements. Mode exactly describes that by its definition, the mean is often close to it for the most common distributions and where it is not, we tend to use other statistics like median or geometric mean instead. Mean could be profoundly affected by outliers or skewed distribution, but in these cases we usually try to deal with them by simply dropping the outliers but we never try to do the opposite, to get rid of the values from the center despite that we aware that the outliers could contain very valuable information. However, their rarity often makes their estimation less reliable.

In medical statistics, outliers have an even a more important role as to some degree diseases are always some non-normal conditions leading to some biomarkers reach very high or very low levels. Of course if our sample contains ill people only these values will not be extreme within our sample, but even the extremes relative to the sample could be very meaningful in terms of the outcomes of the disease.

There is a branch of statistics which focuses on the extreme and - by that nature - rare events. This is called Extreme Value Statistics (EVS) and it is based on Extreme Value Theory (EVT). This originates in the works of Ronald Fisher, Leonard Henry Caleb Tippett and Boris Vladimirovich Gnedenko, the central result of which is summarized in the so-called Fisher-Tippett-Gnedenko theorem [1], [2]. Because these events or observations are rare, and form just a fraction of our total sample - thus have much smaller effective sample size - their analysis is exceptionally difficult.

The EVS is often used in architecture, weather and climate analysis, sports and finance where rare, extreme events have overwhelming impact, but EVS is rarely used in medical statistics. One important reason for this is the lack of data on extremes and the cost of obtaining them compared to natural 
factors like weather which is simple to observe and abundant data are (publicly) available, with daily or even higher frequency for very remote places of Earth and from decades ago. Even for very serious conditions, important biomarkers are usually those that require some sample taken which is typically followed by a complex and expensive process to analyse that; thus it might be measured for patient maybe a couple or dozen times through their clinical history.

Electronic patient monitoring and electronic health records increase the amount of data in medical research as well. The amount of data recorded multiples by each year [3]. Some areas benefit from this more than the others.

Measuring or monitoring blood sugar (glucose) levels became available and more common relatively long time ago [4]. With the introduction of continuous glucose monitoring system (CGMS), even high-frequency (typically 5 minutes sampling time), longer-term (up to a week) measurement became available [5].

Diabetic patients lost the ability to maintain normal glucose levels. Glucose levels that are not extreme to cause acute problems or any symptoms, but are higher than normal can still result in very severe complications such as blindness or the loss of limbs on the long term. Extremely high or low levels of glucose can lead to acute medical emergencies, including the loss of consciousness, onset of coma or even death [6]. To address this problem external control of insulin (and possibly glucagon) - the hormones that control the absorption of glucose - is the best available solution but maintaining their normal level is difficult to achieve as the effects of treatment or any other factors (activity, caloric intake) have a major role in the process and are different from person to person. Real-time control of glucose level is the aim of the Artificial Pancreas (AP) [7] through measuring blood glucose and injecting insulin to the blood stream in a closed loop control fashion. The proper control is still an ongoing issue and there are a lot of different algorithms and developments out there.

The current practice of summarizing continuously measured blood glucose curves involves presenting indicators that mostly focus on variability itself, irrespectively of how extreme the blood glucose level was, thus these are unable to capture the practical performance of the compared algorithms in relation to the risks caused by the extreme glucose levels. The extremities are typically captured only by very crude indicators (such as proportion of time spent in hyper- or hypoglycaemic state).

With the use of EVT we should be able to give an estimate for the probability of blood glucose level exceeding a certain threshold or a return level which represents the period when an extreme occurs once on average and also give confidence intervals (CI) like we do in "regular" statistics. This might catch the essence of the problem better as this would describe the chance of an adverse event of interest such as losing consciousness thus reflecting directly the risks and making the comparison of controllers more relevant.

Our aim is to demonstrate that EVT enables us to charac- terize a blood glucose curve focusing on the more relevant extremes, which can be used - among others - to create patient-level summary metrics and to assess the performance of artificial pancreas algorithms in a more precise and practical way.

Using this theorem, we are able to fit model from the existing data, and then estimate the expected maximum for different time-spans or the return value, with confidence intervals. Practically the maximum we expect to happen once day will be lower than what we expect to happen in every 10 years, but using the above theorem allows us to extrapolate in time (i.e., estimating the maximum in 1 year by using only a month of data).

\section{Methods}

\section{A. Extreme Value Theorem}

There are two main approaches when it comes to the analysis of extreme values and both approaches are relevant in the assessment of the performance of the control of glucose levels.

The Peak Over Threshold (POT) approach uses a cut-off level and takes all values over that level as a secondary sample. This sample would asymptotically follow a Generalised Pareto distribution was first introduced by James Pickands III in 1975 [8].

In contrast, the block maxima (BM) approach, that will be applied in the present paper, splits the data to equal sized blocks and takes only the maximum of each block as a secondary sample to analyse. The behaviour of these extreme values were described by Ronald Fisher and Leonard Henry Caleb Tippett in 1928 [1] and later proven by Boris Vladimirovich Gnedenko in 1943 [2]. Together these form the Fisher-Tippett-Gnedenko theorem which establishes that after taking the secondary sample from the block maxima (renormalization) these converge in distribution to the following distribution:

$$
F(x)=\exp \left[-\left\{1+\xi\left(\frac{x-u}{\sigma}\right)\right\}_{+}^{1 / \xi}\right]
$$

This is called the Generalized Extreme Value (GEV) distribution. It covers three special cases based on the value of $\xi$, which are the following:

1) Frechet distribution $(\xi>0)$ :

$$
F_{\alpha}(x)=\exp \left\{-\left(\frac{x-u}{\sigma}\right)^{-\alpha}\right\}
$$

2) Weibull distribution $(\xi<0)$ :

$$
F(x)=\exp \left\{-\left(-\left(\frac{x-u}{\sigma}\right)^{\alpha}\right)\right\}
$$

3) Gumbel distribution $(\xi=0)$ :

$$
F(x)=\exp \left\{-\exp \left(\frac{x-u}{\sigma}\right)\right\}
$$

The role of extreme value theorem for maxima or for peak over a threshold is very similar to the role of central limit 
theorem for means, except a remarkable difference: the central limit theorem applies to the mean of a sample from any distribution with finite variance but the Fisher-Tippet-Gnedenko theorem is slightly limited. It sates that if the distribution of a normalised maximum converges, then the limit must be one of the distributions described above. It does not contain that the secondary, normalised sample does converge. [9]

\section{B. Patient data}

The sample was taken from type 1 diabetic patient acquired through Freestyle Libre continuous glucose monitoring containing 476 complete days of data acquired with sampling time of 15 minutes. There are 45696 observations in total [10].

\section{Programs used}

The analysis was carried out using the $\mathrm{R}$ statistical program package version 3.6.2 [11] and ExtRemes 2.0 [12] package.

\section{RESUlTS}

The time series of the blood glucose measurements of the sample are shown in Figure 1. The median glucose level across the whole dataset was $135 \mathrm{mg} / \mathrm{dl}$, with a range of 25-429 $\mathrm{mg} / \mathrm{dl}$.

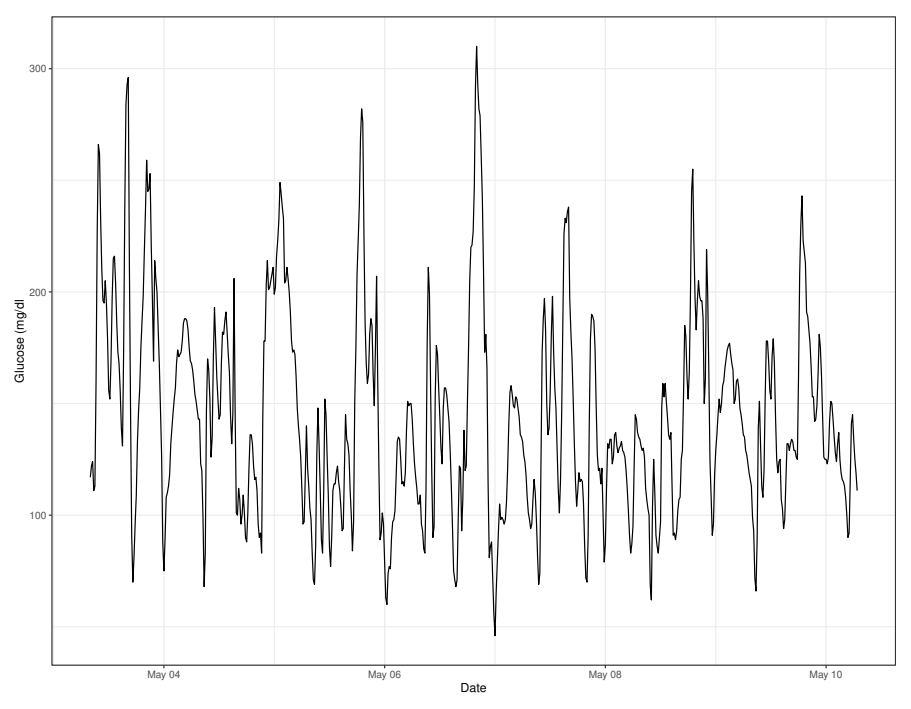

Fig. 1. Plot of a week of glucose measurements

A BM model was fitted to the dataset with the block maximum calculated with a block size of 4 (of 15 minutes long periods, i.e., hourly maxima were calculated). The parameters of the generalised extreme value distribution were: $\mu=133.74$ (95\% CI: $134.64,135.54)$ as location, $\sigma=42.93$ (95\% CI: $43.58,44.23)$ as scale and $\xi=-0.058$ (95\% CI: -0.045 , $0.031)$ as shape parameters. The shape parameter is negative and its CI does not contain 0, so the fitted GEV distribution is a Weibull distribution. (This is also confirmed by using a likelihood ratio test to compare this model to the restricted model with $\xi=0$, i.e., Gumbel model which results in $p<0.0001$.) The goodness of fit was assessed graphically by various diagnostic plots shown on Fig. 2. The model fitted very well to a certain level around $320 \mathrm{mg} / \mathrm{dl}$ and it seems above that the data is slightly less extreme than what the Weibull distribution would suggest, but the overall fit seems fine despite the fact that only a very small fraction of the observations exceeded $270 \mathrm{mg} / \mathrm{dl}(1.97 \%)$.

Results expressed in return levels are summarised in Table I. These are the maximums which occur once on average during the given return period.

TABLE I

RETURN LEVELS

\begin{tabular}{|c|c|c|}
\hline Return level & Estimate $(\mathbf{m g} / \mathbf{d l})$ & $\mathbf{9 5 \%}$ CI $(\mathbf{m g} / \mathbf{d l})$ \\
\hline 1 day & 262.95 & $260.23-265.67$ \\
\hline 1 week & 334.11 & $328.00-340.22$ \\
\hline 28 days & 383.02 & $373.38-392.67$ \\
\hline 1 year & 459.82 & $442.53-477.10$ \\
\hline
\end{tabular}

Using the BM approach we are also able to calculate the probability that the blood glucose level exceeds a certain threshold, as the appropriate quantile of the fitted distribution. (This applies for the whole range of possible values not just for a subset above a certain level like with the Peak Over Threshold (POT) method.)

As an illustration, we chose $270 \mathrm{mg} / \mathrm{dl}$ as one of the limits, as it is within the range of our actual data, but detectable symptoms as cognitive impairment are expected at this level [13] and $600 \mathrm{mg} / \mathrm{dl}$ as a secondary limit which is way beyond the range of the available data, but it is an important physiological limit for diabetic hyperosmolar syndrome which leads to coma for $25-50 \%$ of the patients [14]. According to the BM model presented above there is $3.47 \%$ probability to exceed $270 \mathrm{mg} / \mathrm{dl}$ (which means 30.4 hours per year) and $4.96 \cdot 10^{-7 \%}$ to exceed $600 \mathrm{mg} / \mathrm{dl}$ (which means 15.6 seconds per year in total).

\section{DISCUSSION}

Several metrics are used in clinical practice to describe the variability of a patient's blood glucose levels to characterise the lack of regular control. These include the Mean Amplitude of Glycaemic Excursions (MAGE) [15], the Continuous Net Overall Glycaemic Action (CONGA) [16], simple coefficient of variation or interquartile range of blood glucose levels, or percentage time spent above or below a certain threshold [17].

These metrics, however, mostly focus on overall variability, not specifically on extremities. (I.e., a patient's variabil

ity can be very high, even if the blood glucose level is never in the extreme range, or conversely, the patient can be in extreme range with very low variability.) Thus, these metrics are not appropriate to capture the clinical risks of having extreme glucose level. Those that do capture this aspect are usually very simple indicators (like percentage time spent in extreme range, or the CVGA plot [18] which is essentially the same, but in graphical form), in contrast to which our approach is based on a much more sophisticated statistical foundation. 

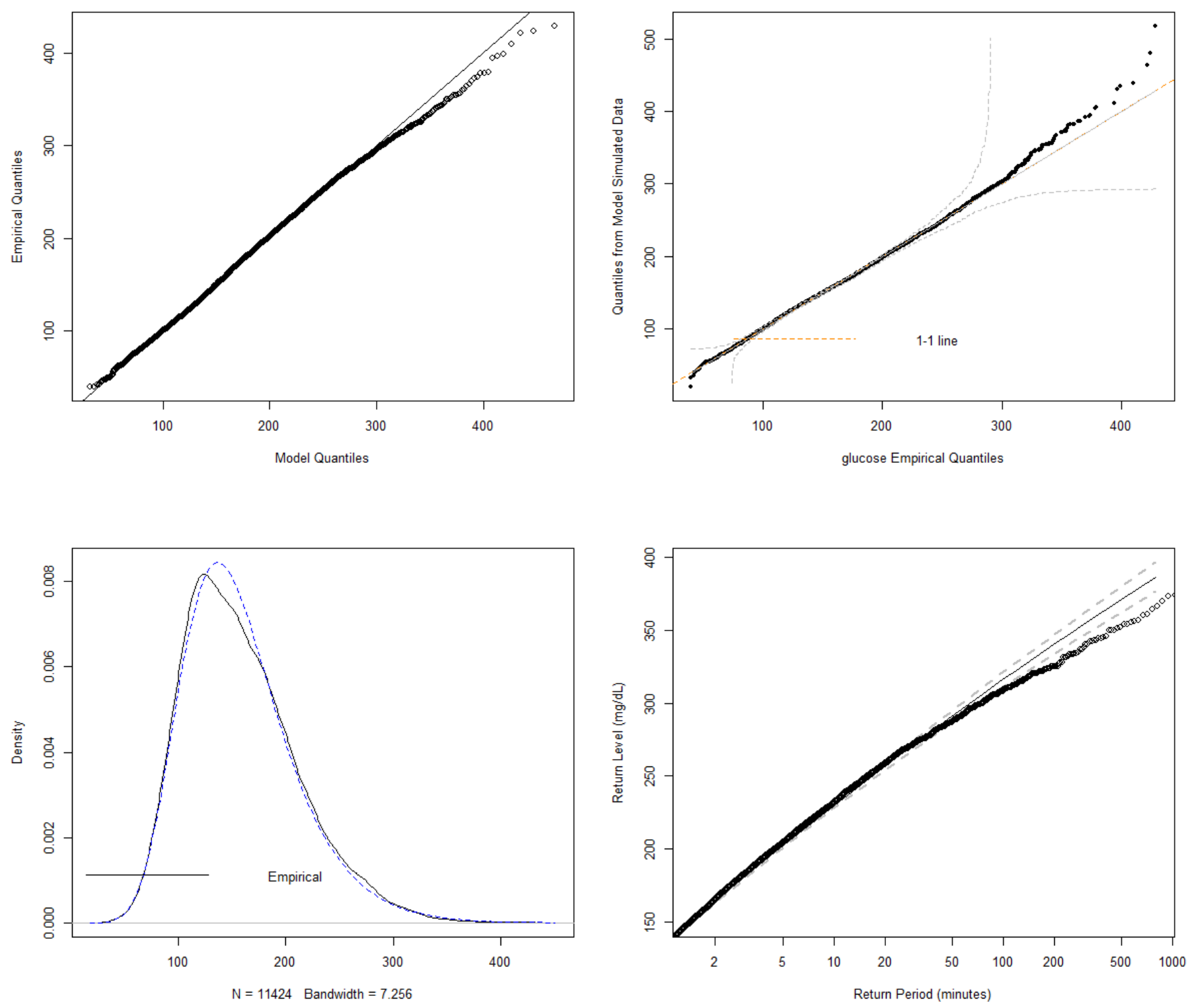

Fig. 2. Model diagnostics plots

Previously in the biomedical field EVT methods like the BM approach were rarely used. Although EVT was used to analyse cholesterol levels [19], pneumonia and influenza deaths [20], the lack off sufficient data is a serious limitation for the EVT in biomedical science.

\section{CONCLUSION}

In this paper the concept of using EVT with BM approach to assess the quality of glucose control based on a CGMS curve been successfully proven. However there are many important points are yet to be answered.

The BM approach reflects to the whole dataset thus the calculated results can directly read as probabilities of reaching certain extremes. This is applicable to compare e.g. different AP controllers comprehensively and also takes into account the extremes occurrence in time as return levels or the directly estimated probabilities, therefore it is also suitable for patientlevel characterization of glycaemia and the associated risks.
The block maxima approach focuses on the distributions of maxima in time so it might be more relevant clinically to give a metric for each patient than the peak over threshold approach.

\section{A. Strengths and weaknesses}

The threshold of diabetic hyperosmolar syndrome (600 $\mathrm{mg} / \mathrm{dl}$ ) was very far from the upper boundaries of the data thus the results very likely to be unreliable for the higher threshold. Yet, we were able to demonstrate the theoretical possibility of calculating estimates beyond the range of the available data. Higher sample sizes would make these estimations more reliable, despite being beyond the range of the data.

Although the other investigated threshold $(270 \mathrm{mg} / \mathrm{dl})$ was not too close to the upper limit of the data $(429 \mathrm{mg} / \mathrm{dl})$ but only the $1.97 \%$ of the observations were above this threshold. According to the model there should be $3.47 \%$ probability for an observation to fall beyond the threshold, but this also 
includes the probability of exceeding the upper limit of the data.

Additionally we did not take into account the dependencies of the measurements: neighbouring or close measurements are strongly correlated because of physiological reasons. A further development could be to extend these methods to be able to account for these correlations in the estimates. Additionally, too low blood glucose levels can also lead to life-threatening conditions thus a natural aspect to extend our investigations to assess not just the peaks but the extremely low measurements.

\section{REFERENCES}

[1] R. A. Fisher and L. H. C. Tippett, "Limiting forms of the frequency distribution of the largest or smallest member of a sample," Mathematical Proceedings of the Cambridge Philosophical Society, vol. 24, no. 2, pp. 180-190, Apr. 1928. [Online]. Available: https://www.cambridge.org/core/journals/mathematicalproceedings-of-the-cambridge-philosophical-society/article/limitingforms-of-the-frequency-distribution-of-the-largest-or-smallest-memberof-a-sample/7BE8DE65FCDFC3ABECFE1054DFB56CB5

[2] B. Gnedenko, "Sur la distribution limite du terme maximum d'une serie aleatoire," Annals of Mathematics, vol. 44, no. 3, pp. 423-453, 1943. [Online]. Available: http://www.jstor.org/stable/1968974

[3] J. K. Wang, D. Ouyang, J. Hom, J. Chi, and J. H. Chen, "Characterizing electronic health record usage patterns of inpatient medicine residents using event log data," PLOS ONE, vol. 14, no. 2, pp. 1-7, 022019. [Online]. Available: https://doi.org/10.1371/journal.pone.0205379

[4] S. Clarke and J. Foster, "A history of blood glucose meters and their role in self-monitoring of diabetes mellitus," British journal of biomedical science, vol. 69, no. 2, pp. 83-93, 2012.

[5] B. W. Bode, "Clinical utility of the continuous glucose monitoring system," Diabetes Technology \& Therapeutics, vol. 2, no. 1, Supplement 1, pp. 35-41, 2000.

[6] J. Wang, D. E. Williams, K. V. Narayan, and L. S. Geiss, "Declining death rates from hyperglycemic crisis among adults with diabetes, us, 1985-2002," Diabetes care, vol. 29, no. 9, pp. 2018-2022, 2006.

[7] C. Cobelli, E. Renard, and B. Kovatchev, "Artificial pancreas: past, present, future," Diabetes, vol. 60, no. 11, pp. 2672-2682, 2011.

[8] J. Pickands III et al., "Statistical inference using extreme order statistics," the Annals of Statistics, vol. 3, no. 1, pp. 119-131, 1975.

[9] A. M. Mood, Introduction to the Theory of Statistics. McGraw-hill, 1950.

[10] S. Contador, gluvarpro: Glucose Variability Measures from Continuous Glucose Monitoring Data, 2019, r package version 2.0. [Online]. Available: https://CRAN.R-project.org/package=gluvarpro

[11] $\mathrm{R}$ Core Team, R: A Language and Environment for Statistical Computing, R Foundation for Statistical Computing, Vienna, Austria, 2019. [Online]. Available: https://www.R-project.org/

[12] E. Gilleland and R. W. Katz, "extRemes 2.0: An extreme value analysis package in R," Journal of Statistical Software, vol. 72, no. 8, pp. 1-39, 2016.

[13] I. Pais, M. Hallschmid, K. Jauch-Chara, S. M. Schmid, K. M. Oltmanns, A. Peters, J. Born, and B. Schultes, "Mood and Cognitive Functions During Acute Euglycaemia and Mild Hyperglycaemia in Type 2 Diabetic Patients," Experimental and Clinical Endocrinology \& Diabetes, vol. 115, no. 01, pp. 42-46, Jan. 2007. [Online]. Available: http://www.thieme-connect.de/DOI/DOI?10.1055/s-2007-957348

[14] F. J. Pasquel and G. E. Umpierrez, "Hyperosmolar Hyperglycemic State: A Historic Review of the Clinical Presentation, Diagnosis, and Treatment," Diabetes Care, vol. 37, no. 11, pp. 3124-3131, Nov. 2014. [Online]. Available: https://www.ncbi.nlm.nih.gov/pmc/articles/PMC4207202/

[15] F. J. Service, G. D. Molnar, J. W. Rosevear, E. Ackerman, L. C. Gatewood, and W. F. Taylor, "Mean amplitude of glycemic excursions, a measure of diabetic instability," Diabetes, vol. 19, no. 9, pp. 644-655, 1970. [Online]. Available: https://diabetes.diabetesjournals.org/content/19/9/644
[16] C. McDonnell, S. Donath, S. Vidmar, G. Werther, and F. Cameron, "A novel approach to continuous glucose analysis utilizing glycemic variation," Diabetes Technology \& Therapeutics, vol. 7, no. 2, pp. 253-263, 2005, pMID: 15857227. [Online]. Available: https://doi.org/10.1089/dia.2005.7.253

[17] G. Marics, Z. Lendvai, C. Lódi, L. Koncz, D. Zakariás, G. Schuster, B. Mikos, C. Hermann, A. J. Szabó, and P. Tóth-Heyn, "Evaluation of an open access software for calculating glucose variability parameters of a continuous glucose monitoring system applied at pediatric intensive care unit," Biomedical engineering online, vol. 14, no. 1, p. 37, 2015.

[18] L. Magni, D. M. Raimondo, C. D. Man, M. Breton, S. Patek, G. De Nicolao, C. Cobelli, and B. P. Kovatchev, "Evaluating the efficacy of closed-loop glucose regulation via control-variability grid analysis," Journal of diabetes science and technology, vol. 2, no. 4, pp. 630-635, 2008.

[19] P. Bermudez and Z. Mendes, "Extreme value theory in medical sciences: Modeling total high cholesterol levels," Journal of statistical theory and practice, vol. 6, pp. 468-491, 092012.

[20] M. Thomas, M. Lemaitre, M. L. Wilson, C. Viboud, Y. Yordanov, H. Wackernagel, and F. Carrat, "Applications of extreme value theory in public health," PloS one, vol. 11, no. 7, 2016. 
M. Szigeti et al. The use of block maxima method of extreme value statistics to characterise blood glucose curves

000438

Authorized licensed use limited to: University of Liverpool. Downloaded on July 27,2020 at 20:40:51 UTC from IEEE Xplore. Restrictions apply. 